\title{
BMJ Open Nutraceuticals and nutritional supplements for the treatment of bipolar disorder: protocol for a systematic review
}

\author{
Melanie M Ashton, ${ }^{1,2,3}$ Michael Berk, ${ }^{1,3,4,5,6}$ Chee H Ng, ${ }^{2}$ Malcolm Hopwood,,${ }^{4,7}$ \\ Bianca Kavanagh, ${ }^{1}$ Lana J Williams, ${ }^{1}$ Jerome Sarris, ${ }^{2,8}$ Olivia M Dean ${ }^{1,3,4}$
}

To cite: Ashton MM, Berk M $\mathrm{Ng} \mathrm{CH}$, et al. Nutraceuticals and nutritional supplements for the treatment of bipolar disorder: protocol for a systematic review. BMJ Open 2019;9:e025640. doi:10.1136/ bmjopen-2018-025640

- Prepublication history and additional material for this paper are available online. To view these files, please visit the journal online (http://dx.doi. org/10.1136/bmjopen-2018025640).

Received 25 July 2018 Revised 14 February 2019 Accepted 20 February 2019
(A) Check for updates

(C) Author(s) (or their employer(s)) 2019. Re-use permitted under CC BY-NC. No commercial re-use. See rights and permissions. Published by BMJ.

For numbered affiliations see end of article.

Correspondence to

Melanie M Ashton;

m.ashton@deakin.edu.au

\section{ABSTRACT}

Introduction First line pharmacological treatments for bipolar disorder (BD) can leave shortfalls in recovery leading to patients seeking alternative and adjunctive treatments such as nutraceuticals. This protocol for a systematic review and proposed meta-analysis aims to answer the research question: in patients with $\mathrm{BD}$, how does use of nutraceutical treatments compare with placebo in reducing depressive and mania symptoms? Methods and analysis Clinical trials will be identified through database searches using PubMed via PubMed, EMBASE via embase.com, Cochrane Central Register of Controlled Clinical Trials (CENTRAL) via cochranelibrary. com and CINAHL Complete via EBSCO. Search terms for $\mathrm{BD}$ and specific nutraceuticals (75 total search terms) will be used. Double-blind, randomised, controlled, clinical trials of adults with $\mathrm{BD}$ will be included in the review. Risk of bias will be assessed using the Cochrane Collaboration's tool for assessing risk of bias in randomised trials.

Ethics and dissemination This review will only look at published data (already reviewed for ethical compliance); therefore, ethical approval is not required. We aim to publish the systematic review in a peer-reviewed journal and present at conferences.

PROSPERO registration number CRD42019100745.

\section{INTRODUCTION}

\section{Bipolar disorder}

Bipolar disorder (BD) is a biphasic disorder characterised by manic and depressive episodes. ${ }^{1}$ Common pathways to pathology include dysregulation of monoamines, increased oxidative stress, perturbed inflammatory processes and mitochondrial disturbances. ${ }^{2}$ The depressive phase of the disorder is more common than mania (3:1) and can be harder to treat. ${ }^{3}$ Most first-line treatments for $\mathrm{BD}$ are more effective at treating the manic phase, including mood stabilisers (eg, lithium) and second-generation antipsychotics (eg, lurasidone or quetiapine). ${ }^{4}$ Antidepressants, especially tricyclic antidepressants and selective serotonin-norepinephrine reuptake
Strengths and limitations of this study

- This review will update and extend previously completed systematic reviews of the effects of nutraceuticals in bipolar disorder (BD).

- This review will employ rigorous screening and assessment of studies to be included allowing for only good quality, peer reviewed publications.

- A strength of this review is the range of potential nutraceutical agents to be assessed.

- A limitation of the review will be the inherent differences in manic and depressive phases means it will be difficult to compare agents across BD as a whole.

- There is likely to be high heterogeneity for the nutraceutical agents and measures for symptomology across the studies limiting the comparisons for a meta-analysis.

inhibitors, can cause some people with BD to switch to a manic phase, therefore antidepressants are not recommended as monotherapy. ${ }^{5-7}$ Other common side effects of currently available treatments for BD can include extrapyramidal symptoms such a tremors, akathisia ${ }^{8}$ and weight gain. ${ }^{89}$ Thus, polypharmacy is more common practice than monotherapy. ${ }^{4}$ Due to tolerability issues and shortfalls in recovery, additional treatment options are often sought.

Nutraceuticals have been defined as functional foods that treat or prevent a disease or disorder ${ }^{10}$ and in particular are nutrients that have been standardised and of pharmaceutical grade. ${ }^{11}$ Originally described as a combination between nutrition and pharmaceuticals, ${ }^{12}$ nutraceuticals are a growing field of medications. The Therapeutic Goods Association of Australia regulate complementary medicines defined as therapeutic goods containing one or more of the following active ingredients: amino acid, mineral, vitamin or provitamin, choline salt, 
lipid (including an essential fatty acid or phospholipid), a sugar, polysaccharide or carbohydrate, mucopolysaccharide, plant or herbal material, an essential oil, charcoal, micro-organism, non-human animal material, or homeopathic preparation. ${ }^{13}$ Dietary supplements have been defined by the Food and Drug Administration in the Dietary Supplement and Health Education Act as an orally administered dietary ingredient that could contain minerals, vitamins, enzymes, amino acids or herbs. ${ }^{14}$ Approximately $30 \%$ of individuals with BD have reported taking nutraceuticals, although the authors highlighted that under-reporting of nutraceuticals is common due to the perceived idea they are often considered relatively benign. Because of this perception of nutraceuticals, treating physicians may not be notified of the patient's nutraceutical use. ${ }^{15}$

The term Nutraceutical encompasses a broad range of agents, which may have differing effects within the body on the pathophysiology of some disorders. Some nutraceuticals have bioactive properties that can target the putative pathophysiology of BD. For example, omega-3 is known for its high anti-inflammatory properties, L-theanine for its antioxidant properties and the amino acid N-acetylcysteine is known for both its anti-inflammatory, antioxidative and mitochondrial properties. We will present an overview of nutraceuticals broadly and then explore subgroups, based on those reported in the previous systematic review to allow comparisons with the previous literature (eg, fatty acids). For the purposes of this review, lithium will not be included despite coming under the definition of minerals. Lithium will not be included as it has been extensively researched previously ${ }^{16}$ and will fall outside of the scope of this review. Two previous systematic reviews have been conducted in this field (Sarris $e t$ $a l^{17}$ and Sylvia $e t a l^{18}$ ). However, given the expansion of the field and progress in the understanding of the underlying biological processes, this systematic review will form a comprehensive and much needed update of the current literature.

\section{Objectives}

The objectives of this systematic review are to identify and evaluate published clinical trials of nutraceuticals as treatments for BD. The systematic review will include both mania and depressive phases of the disorder. However, as the depressive phase of the disorder is the most frequent and disabling phase and therefore been more widely studied, it is likely to return the most results. If there is sufficient data within two studies or more and homogeneity established, a meta-analysis will be conducted to assess the effect of nutraceuticals compared with placebo. If the studies demonstrate considerable heterogeneity then subanalyses will be performed. The aim of this review will be to answer the research question: In patients with $\mathrm{BD}$, how does use of nutraceutical treatments compare with placebo in reducing symptoms of depression and mania?

\section{METHODS}

\section{Search methods}

This systematic review protocol has been prepared in accordance with Preferred Reporting Items for Systematic Reviews and Meta-Analysis (PRISMA) Protocols guidelines. ${ }^{19}$

Relevant literature will be identified via electronic searches using PubMed via PubMed, EMBASE via embase. com, Cochrane Central Register of Controlled Clinical Trials (CENTRAL) via cochranelibrary.com and CINAHL Complete via EBSCO. Searches will be conducted from inception to the date of search. In addition to the database searches, reference lists of other systematic reviews and retrieved trials will also be reviewed for studies to be included in the screening process.

\section{Search strategy}

PICO (Patient, Intervention, Comparison/Control, Outcome) search framework was used to develop search terms relating to $\mathrm{BD}$ and nutraceuticals. Key search terms will be used as follows, by searching 'all fields'. Relevant formatting for each database will be used. The following Medical Subject Headings (MeSH) and Emtree terms will be used ('bipolar disorder' OR 'bipolar and related disorders' OR 'cyclothymic disorder' OR 'bipolar I disorder' OR 'bipolar II disorder') AND (prebiotics OR probiotics OR 'nutritional supplement' OR 'nutrition supplement' OR 'nutrition therapy' OR 'nutritional support' OR minerals OR mineral OR 'dietary supplement' OR 'complementary therapies' OR 'alternative medicine' OR 'medicine, traditional' OR 'traditional medicine' OR 'amino acids' OR acetylcysteine OR 'fatty acids' OR 'fatty acid' OR 'eicosapentaenoic acid' OR 'fatty acid, omega-3' OR 'Fatty acid, omega-6' OR 'folic acid' OR antioxidants OR 'fatty acid, essential' OR tryptophan OR creatine OR chromium OR inositol OR choline OR zinc OR ubiquinone OR curcumin OR 'thioctic acid' OR acetylcarnitine OR s-adenosylmethionine OR melatonin OR taurine) AND ('clinical trial' OR 'randomized controlled trial' OR 'controlled clinical trial'). In addition, other common, non-MeSH key terms will be included in the coinciding sections of the search: ('bipolar affective disorder' OR 'bipolar depression' OR 'bipolar mania' OR mania OR hypomania OR cyclothymia OR BPAD OR BD) AND (Nutraceutical OR 'functional food' OR nutrient* OR supplement OR 'nutrient-based therapy' OR complementary OR vitamin* OR 'amino acid' OR n-acetylcysteine OR methylfolate OR PUFA OR cannabinoids OR omega* OR folate OR magnesium OR tryptophan OR 'essential fatty acids' OR tonic OR 'coenzyme q10' OR 'alpha-lipoic acid') AND (RCT OR trial OR 'randomised controlled trial'). The full search strategy for each database can be found in online supplementary file 1 .

\section{Inclusion/exclusion criteria \\ Types of studies}

For this review, only peer-reviewed, published, double-blind, randomised, controlled trials will be included. Studies may either include adjunctive agents or monotherapy and will be compared with either a placebo or other intervention. 
Only studies testing the effects of the medication on the reduction of depressive or mania symptoms of $\mathrm{BD}$ will be included. Therefore, prophylaxis studies and studies will be excluded. Case reports, observational studies, open-label trials, cross-sectional design studies, grey literature, protocol papers and conference presentations will also be excluded.

\section{Types of participants}

We will review studies including adults with a diagnosis of BD I, II, NOS based on Diagnostic and Statistical Manual of Mental Disorders (DSM) or International Classification of Diseases (ICD) criteria. In-patient and out-patient participants will all be included.

Subgroup analyses will be performed to determine the effects of the study medication specifically on the active depressive phase of BD. For the relevant studies, current depression must be assessed using the following standardised scales: Montgomery Åsberg Depression Rating Scale (MADRS), ${ }^{20}$ Hamilton Depression Rating Scale (HAM-D), ${ }^{21}$ Hospital Anxiety and Depression Scale (HADS) ${ }^{22}$ Bipolar Depression rating scale (BDRS), ${ }^{23}$ Beck Depression Inventory ${ }^{24}$ or Quick Inventory of Depressive Symptomatology, Patient Health Questionnaire-9. ${ }^{25}$ Mania must be assessed using the following standardised scales: Young Mania Rating Scale (YMRS), ${ }^{26}$ The Bech-Fafaelsen Mania Rating Scale, ${ }^{27}$ the mania subscale of the Schedule for Affective Disorders and Schizophrenia ${ }^{28} 29$ and the Altman Self-Rating Mania Scale. ${ }^{30}$ Appropriate cut-off scores will be used for relevant severity of disorder in each study, as determined by or established for each scale. For example, if a study was interested in at least moderate level of depression and utilising the MADRS, a cut-off score of 20 or above would be appropriate given 0-6 is considered recovered, 7-19 mild depression, 20-34 moderate depression and 35-60 severe depression. ${ }^{31}$

\section{Types of interventions}

To be included in the review, interventions must come under the definition of nutraceuticals, complementary medicines or dietary supplements (see the Introduction section for definitions) and must aim to improve depressive or manic symptoms. Following the initial search, a table of agents which are included and excluded through the systematic review process will be provided. Comparator arms of the studies may be placebo or another intervention (eg, another medication).

\section{Outcome measures}

Primary outcome measure will be an assessment of depression or mania symptomology (eg, standardised mean differences in scores on MADRS, BDRS, YMRS and so on). In the case of multiple outcome measures in the study, only the primary outcome will be included in the systematic review and potential meta-analysis. Any secondary outcomes will not be included as they are likely to be underpowered.

\section{Patient and public involvement}

Participants and the public were not involved in the design of the review. As the review will use only previously published research, consent was not required from participants and dissemination to participants will not be required. The authors are working towards better outcomes for participants with $\mathrm{BD}$ and factors that influence those outcomes were considered when designing this review.

\section{DATA ANALYSIS}

\section{Data management}

Covidence, ${ }^{32}$ an online database tool, will be used to manage references and search results during the screening and reviewing process. The program allows for handling of duplicate records and detailed tracking of inclusion/exclusion of references allowing for easy extraction into PRISMA flow diagrams.

\section{Identification of eligible studies}

The initial search will be conducted by one lead reviewing author to identify relevant articles. Articles will be screened for inclusion/exclusion based on title and abstract; then by reviewing the full text. A second independent reviewing author will independently screen the titles and abstracts. If there are any discrepancies, an experienced third author will act as adjudicator to decide on inclusion into full-text review. After screening, relevant full-text articles will be independently reviewed for bias and content by two independent reviewing authors. If there are discrepancies or disagreements between the two reviewing authors and a consensus cannot be reached, a third independent reviewing author will adjudicate.

\section{Assessment of included articles}

Extracted data will include study design, details of intervention(s), details of comparator arm (ie, placebo or intervention), outcome measures, characteristics of samples and risk of bias. The extracted data will follow Consolidated Standards of Reporting Trials (CONSORT) guidelines. ${ }^{33}$ STATA Data Analysis and Statistical Software V.15, ${ }^{34}$ will be used to analyse data and data synthesis. Treatment effects will be measured by standardised mean differences with $95 \%$ CI to allow for differences in interpretation of scores across the scales. In this model, sample sizes and standard deviations will be used to give weightings to each study. If there are no clear summaries of all data to fulfil the CONSORT guidelines and risk of bias then in the first instance authors will be contacted in an attempt to fill in gaps. If contacting the author is not possible then the studies will not be included due to insufficient data.

The Cochrane Collaboration's tool for assessing risk of bias in randomised trials ${ }^{35}$ will be used to assess bias of all included trials. The criteria for bias included scores of 'low risk of bias', 'high risk of bias' and 'unknown risk of bias' in the following domains: random sequence generation, allocation concealment, blinding, incomplete outcome data, selective reporting and other bias. In the event of missing data in the published study, published protocols will also be used to assess bias, such as publication bias. Publication bias will be assessed via funnel plots. Attempts 
will be made to contact corresponding authors for missing data in the published studies. In accordance with the Cochrane Handbook $5.1^{36}$ suggestions, $\mathrm{I}^{2}$ will be used to assess for heterogeneity of the trials. If $\mathrm{I}^{2}$ is $>50 \%$ then the studies will be considered to have a substantial heterogeneity and will be included in the summarising sections of the systematic review, but the proposed meta-analysis will not be conducted. Quality of evidence of the studies will be assessed and summarised using the Grades of Recommendation, Assessment, Development Evaluation approach. ${ }^{37}$ If a meta-analysis is possible, a random-effects model will be used. Results will be reported as standardised mean differences with $95 \%$ CI.

\section{Subgroup analysis}

The following subgroup analyses will be performed if there is significant heterogeneity across the studies (if $\mathrm{I}^{2}>50 \%$ ). Subgroup analyses will include different phases of BD and different groups of nutraceuticals.

1. Effect of treatment on depressive symptoms.

2. Effect of treatment on mania symptoms.

3. Depressive phase compared with euthymia.

4. Any phase of BD upon entry into study and depression or mania scores at treatment phase completion.

5. Treatment outcomes of vitamins.

6. Treatment outcomes of fatty acid supplements (eg, omega-3).

7. Treatment outcomes of minerals (eg, zinc, magnesium).

8. Treatment outcomes of amino acids.

9. Treatment outcomes of individual agents.

10. Combination treatments (eg, multivitamins and minerals) versus single treatments.

\section{Sensitivity analysis}

Sensitivity analyses will be conducted to take into consideration the following methodological differences (1) differences in diagnostic tool (DSM vs ICD), (2) difference in BD subgroups, (3) different measures of symptoms (eg, MADRS vs HADs) and (4) length of study. For each sensitivity analysis data will be analysed separately and results will be compared with the initial all-inclusive analysis to ensure similar results. In addition, studies will be analysed for differences in gender, age, location and dose of nutraceutical studied, if there are differing doses. If data for the sensitivity analysis is missing in the studies, authors will be contacted in the first instance. If more details cannot be obtained, the study will not be included in the analysis and this will be noted in the results.

\section{Presentation and reporting of results}

The review will adhere to PRISMA guidelines and data will be reported using a PRISMA flow diagram. ${ }^{38}$ The PRISMA flow diagram will depict numbers excluded at each stage of screening (identification, screening, eligibility and reasons for exclusion) to show the number of studies included and excluded in the review.

\section{Ethics and dissemination}

This review will only use published data that has received ethical approval, therefore specific ethical approval for this review is not required. We aim to publish the systematic review in a peer-reviewed journal and the results may be presented at a scientific conference.

\section{CONCLUSION}

This rigorous review will evaluate the current literature on the use of nutraceuticals as treatments for BD. This review may be used by clinicians to assist with treatment choices for their patients with BD. Ultimately, this review aims to evaluate additional treatment options for $\mathrm{BD}$ and if positive, with the view to reduce the burden of the disorder.

\section{Author affiliations}

${ }^{1}$ IMPACT Strategic Resarch Centre, School of Medicine, Deakin University, Geelong, Victoria, Australia

2Professorial Unit, The Melbourne Clinic, Department of Psychiatry, University of Melbourne, Richmond, Victoria, Australia

${ }^{3}$ Florey Institute for Neuroscience and Mental Health, University of Melbourne, Parkville, Victoria, Australia

${ }^{4}$ Department of Psychiatry, The University of Melbourne, Royal Melbourne Hospital, Parkville, Victoria, Australia

${ }^{5}$ Orygen, The National Centre of Excellence in Youth Mental Health, Parkville, Victoria, Australia

${ }^{6}$ Centre of Youth Mental Health, The University of Melbourne, Parkville, Victoria, Australia

${ }^{7}$ Professorial Psychiatry Unit, The Albert Road Clinic, University of Melbourne, Melbourne, Victoria, Australia

${ }^{8}$ NICM Health Research Institute, Western Sydney University, Westmead, New South Wales, Australia

Acknowledgements The authors wish to thank Blair Kelly, Deakin University, for advice on the search strategy and library support.

Contributors MMA conceptualised and designed the research questions and search strategy, prepared the manuscript draft, edited and approved the final version of the manuscript. MB conceptualised the research question, revised the search strategy and edited and approved the manuscript. CHN conceptualised the research question, revised the search strategy and edited and approved the manuscript. MH conceptualised the research question, revised the search strategy and edited and approved the manuscript. BK revised the search strategy, edited and approved the manuscript. LJW revised the search strategy, edited and approved the manuscript. JS revised the search strategy, edited and approved the manuscript. OMD conceptualised the research question and search strategy, edited the manuscript and approved the final version.

Funding The authors would like to acknowledge the NHMRC Project Grant Scheme (APP1121510) for supporting this review. MMA would further like to acknowledge the support of Australian Rotary Health/lan Parker Bipolar Research Fund PhD scholarship and the ASBDD/Lundbeck PhD neuroscience scholarship. MB is supported by a NHMRC Senior Principal Research Fellowship (APP1059660). BK is supported by Deakin University, the Australian Government Research Training Program Scholarship and Australian Rotary Health. LJW is supported by a NHMRC Career Development Fellowship (APP1064272). JS is supported by an NHMRC Clinical Research Fellowship (APP1125000). OMD is supported by a NHMRC R.D. Wright Biomedical Research Fellowship (APP1145634). No funding bodies had any role in developing the protocol.

Competing interests MMA has received grant/research support from Deakin University, Australasian Society for Bipolar Depressive Disorders, Lundbeck, Australian Rotary Health, lan Parker Bipolar Research Fund and Cooperative Research Centre for Mental Health. MB has received grant support from NIH, Simons Autism Foundation, Cancer Council of Victoria, CRC for Mental Health, Stanley Medical Research Foundation, MBF, NHMRC, Beyond Blue, Geelong Medical Research Foundation, Bristol Myers Squibb, Eli Lilly, GlaxoSmithKline, Organon, Novartis, Mayne Pharma and Servier. MB has received Grant/Research Support 
from the NIH, Cooperative Research Centre, Simons Autism Foundation, Cancer Council of Victoria, Stanley Medical Research Foundation, MBF, NHMRC, Beyond Blue, Rotary Health, Meat and Livestock Board, Astra Zeneca, Woolworths, Avant and the Harry Windsor Foundation, book royalties from Oxford University Press, Cambridge University Press, Springer Nature and Allen and Unwin, has been a speaker for Astra Zeneca, Lundbeck, Merck and Servier and served as a consultant to Allergan, Astra Zeneca, Bioadvantex, Bionomics, Collaborative Medicinal Development, Grunbiotics, Janssen Cilag, LivaNova, Lundbeck, Merck, Mylan, Otsuka and Servier. MB is a coinventor on two provisional patents regarding the use of NAC and related compounds for psychiatric indications, assigned to the Mental Health Research Institute. MB is a coinventor on a patent application regarding the use of mangosteen and related compounds for psychiatric indications, assigned to Deakin University. CHN had served in the Servier, Janssen-Cilag, Wyeth and Eli Lilly Advisory Boards, received research grant support from Wyeth and Lundbeck, and speaker honoraria from Servier, Lundbeck, Bristol-Myers Squibb, Organon, Eli Lilly, GlaxoSmithKline, Janssen- Cilag, Astra-Zenaca, Wyeth, and Pfizer. MH has received grant support from ISSCR, Servier, US DOD and Bionomics, has been a speaker for Janssen-Cilag, Lundbeck, and Servier, and has been a consultant for AstraZeneca, Eli Lilly, Janssen-Cilag, Lundbeck and Servier. BK has received research support from Deakin University, the Australian Government Research Training Program Scholarship and Australian Rotary Health. LJW has received Grant/Research support from Eli Lilly, Pfizer, The University of Melbourne, Deakin University and the NHMRC. JS has received either presentation honoraria, travel support, clinical trial grants, book royalties or independent consultancy payments from: Integria Healthcare \& MediHerb, Pfizer, Scius Health, Key Pharmaceuticals, Taki Mai, FIT-BioCeuticals, Blackmores, Soho-Flordis, Healthworld, HealthEd, HealthMasters, Kantar Consulting, Research Reviews, Elsevier, Chaminade University, International Society for Affective Disorders, Complementary Medicines Australia, SPRIM, Terry White Chemists, ANS, Society for Medicinal Plant and Natural Product Research, Sanofi-Aventis, Omega-3 Centre, the National Health and Medical Research Council, CR Roper Fellowship. OMD is a R.D. Wright Biomedical Research Fellow and has received grant support from the Brain and Behavior Foundation, Simons Autism Foundation, Stanley Medical Research Institute, Deakin University, Lilly, NHMRC and Australasian Society for Bipolar and Depressive Disorders (ASBDD)/Servier

Patient consent for publication Not required.

Provenance and peer review Not commissioned; externally peer reviewed.

Open access This is an open access article distributed in accordance with the Creative Commons Attribution Non Commercial (CC BY-NC 4.0) license, which permits others to distribute, remix, adapt, build upon this work non-commercially, and license their derivative works on different terms, provided the original work is properly cited, appropriate credit is given, any changes made indicated, and the use is non-commercial. See: http://creativecommons.org/licenses/by-nc/4.0/.

\section{REFERENCES}

1. American Psychiatric Association (APA). Diagnostic and statistical manual of mental disorders, fifth edition (DSM-5). Arlington, VA: APA, 2013.

2. Data-Franco J, Singh A, Popovic D, et al. Beyond the therapeutic shackles of the monoamines: new mechanisms in bipolar disorder biology. Prog Neuropsychopharmacol Biol Psychiatry 2017;72:73-86.

3. Judd LL, Akiskal HS, Schettler PJ, et al. The long-term natural history of the weekly symptomatic status of bipolar I disorder. Arch Gen Psychiatry 2002;59:530-7.

4. Malhi GS, Bassett D, Boyce P, et al. Royal Australian and New Zealand college of psychiatrists clinical practice guidelines for mood disorders. Aust N Z J Psychiatry 2015;49:1087-206.

5. Sachs GS, Nierenberg AA, Calabrese JR, et al. Effectiveness of adjunctive antidepressant treatment for bipolar depression. $N$ Engl $J$ Med 2007;356:1711-22.

6. Daray FM, Thommi SB, Ghaemi SN. The pharmacogenetics of antidepressant-induced mania: a systematic review and metaanalysis. Bipolar Disord 2010;12:702-6.

7. Yatham LN, Kennedy SH, Parikh SV, et al. Canadian Network for Mood and Anxiety Treatments (CANMAT) and International Society for Bipolar Disorders (ISBD) collaborative update of CANMAT guidelines for the management of patients with bipolar disorder: update 2013. Bipolar Disord 2013;15:1-44.

8. Serretti A, Chiesa A, Calati R, et al. Side effects associated with psychotropic medications in patients with bipolar disorder: evidence from two independent samples. J Psychopharmacol 2013;27:616-28.
9. Malhi GS, Mitchell PB, Caterson I. 'Why getting fat, Doc?' Weight gain and psychotropic medications. Aust N Z J Psychiatry 2001;35:315-21.

10. Kalra EK. Nutraceutical-definition and introduction. Aaps Pharmsci 2003;5:27-8.

11. Sarris J, Murphy J, Mischoulon D, et al. Adjunctive nutraceuticals for depression: a systematic review and meta-analyses. Am J Psychiatry 2016;173:575-87.

12. DeFelice SL. The nutraceutical revolution: its impact on food industry R\&D. Trends in Food Science and Technology 1995;6:59-61.

13. Therapeutic Goods Administration (TGA). Therapeutic goods regulations. 1990 https://www.legislation.gov.au/Details/ F2018C00193 (Accessed 2nd July 2018).

14. National Institute of Health. Dietary health and supplement act. 1994 https://ods.od.nih.gov/About/dshea_wording.aspxv (Accessed 2nd July 2018).

15. Bauer M, Glenn T, Conell J, et al. Common use of dietary supplements for bipolar disorder: a naturalistic, self-reported study. Int J Bipolar Disord 2015;3:29.

16. Licht RW. Lithium: still a major option in the management of bipolar disorder. CNS Neurosci Ther 2012;18:219-26.

17. Sarris J, Lake J, Hoenders R. Bipolar disorder and complementary medicine: current evidence, safety issues, and clinical considerations. J Altern Complement Med 2011;17:881-90.

18. Sylvia LG, Peters AT, Deckersbach T, et al. Nutrient-based therapies for bipolar disorder: a systematic review. Psychother Psychosom 2013;82:10-19.

19. Shamseer L, Moher D, Clarke M, et al. Preferred reporting items for systematic review and meta-analysis protocols (PRISMA-P) 2015: elaboration and explanation. BMJ 2015;349:g7647.

20. Montgomery SA, Asberg M. A new depression scale designed to be sensitive to change. Br J Psychiatry 1979;134:382-9.

21. Hamilton M. Development of a rating scale for primary depressive illness. Br J Soc Clin Psychol 1967;6:278-96.

22. Zigmond AS, Snaith RP. The hospital anxiety and depression scale. Acta Psychiatr Scand 1983;67:361-70.

23. Berk M, Dodd S, Dean OM, et al. The validity and internal structure of the bipolar depression rating scale: data from a clinical trial of $\mathrm{n}$-acetylcysteine as adjunctive therapy in bipolar disorder. Acta Neuropsychiatr 2010;22:237-42.

24. Beck AT, Steer RA, Brown GK. Beck depression inventory-II. San Antonio, TX: Psychological Corpororation, 1996.

25. Kroenke K, Spitzer RL, Williams JB. The phq-9. J Gen Intern Med 2001;16:606-13.

26. Young RC, Biggs JT, Ziegler VE, et al. A rating scale for mania: reliability, validity and sensitivity. Br J Psychiatry 1978;133:429-35.

27. Bech P, Bolwig TG, Kramp P, et al. The bech-rafaelsen mania scale and the hamilton depression scale. Acta Psychiatr Scand 1979;59:420-30.

28. Johnson MH, Magaro PA, Stern SL. Use of the SADS--C as a diagnostic and symptom severity measure. J Consult Clin Psychol 1986;54:546-51.

29. Rogers R, Jackson RL, Salekin KL, et al. Assessing Axis I symptomatology on the SADS-C in two correctional samples: the validation of subscales and a screen for malingered presentations. $J$ Pers Assess 2003;81:281-90.

30. Altman EG, Hedeker D, Peterson JL, et al. The altman self-rating mania scale. Biol Psychiatry 1997;42:948-55.

31. Snaith RP, Harrop FM, Newby DA, et al. Grade scores of the montgomery-asberg depression and the clinical anxiety scales. $\mathrm{Br} \mathrm{J}$ Psychiatry 1986;148:599-601.

32. Veritas Health Innovation. Covidence systematic review software [program]. Melbourne, Australia: Veritas Health Innovation. www. covidence.org

33. Schulz KF, Altman DG, Moher D. CONSORT 2010 statement: updated guidelines for reporting parallel group randomised trials. BMJ 2010;340:c332.

34. StataCorp LLC. Stata statistical software: release 15. [program]. 15 version. College Station, TX: StataCorp LLC.

35. Higgins JP, Altman DG, Gøtzsche PC, et al. The Cochrane Collaboration's tool for assessing risk of bias in randomised trials. BMJ 2011;343:d5928.

36. Higgins J, Altman D, Sterne J. Chapter 8: Assessing risk of bias in included studies. In: Higgins JPT, Green S, Cochrane handbook for systematic reviews of interventions Version 5.1. 0, 2011.

37. Guyatt GH, Oxman AD, Vist GE, et al. GRADE: an emerging consensus on rating quality of evidence and strength of recommendations. BMJ 2008;336:924-6.

38. Moher D, Liberati A, Tetzlaff J, et al. Preferred reporting items for systematic reviews and meta-analyses: the PRISMA statement. PLoS Med 2009;6:e1000097. 